\title{
Data mining models for predicting oral cancer survivability
}

\author{
Neha Sharma $\cdot$ Hari Om
}

Received: 8 July 2013/Revised: 18 September 2013/Accepted: 16 October 2013/Published online: 31 October 2013

(C) Springer-Verlag Wien 2013

\begin{abstract}
In this paper, three predictive models are proposed to identify the most effective model for predicting the survival rate of oral cancer in patients who visit the ENT OPD. This study examined 1,024 patients who visited a tertiary care center during Jan 2004 and Dec 2009. The predictive models developed in this work are Single Tree, Decision Tree Forest and TreeBoost based on classification analysis. For all these models, it is observed that there is no misclassified row in any category and all cases have correctly been classified. The sensitivity and specificity of these models is $100 \%$. All the models display similar results and performance; however, as the TreeBoost model considers all 18 predictors for each split, it is marginally better than the Single Tree and Decision Tree Forest. The experimental results of probability calibration, threshold analysis and lift-gain are also slightly better in case of the TreeBoost model. Thus, the TreeBoost classification model is optimal for predicting survivability of oral cancer patients.
\end{abstract}

Keywords Oral cancer - Data mining - Single tree . Decision tree forest . TreeBoost - Classification . Early detection $\cdot$ Prevention

\footnotetext{
N. Sharma $(\bowtie)$

Padmashree Dr. D.Y. Patil Institute of Master of Computer Applications, University of Pune, Akurdi, Pune 411044, Maharashtra, India

e-mail: nvsharma@rediffmail.com

H. Om

Computer Science and Engineering Department, Indian School of Mines, Dhanbad, Jharkhand, India

e-mail: hari.om.cse@ismdhanbad.ac.in
}

\section{Introduction}

Every year approximately 2,00,000 deaths worldwide and 46,000 deaths particularly in India account for oral cancer (Jemal et al. 2010). The study shows that the developing countries like Melanesia, South-Central Asia and Central and Eastern Europe have the highest rate of oral cavity cancer, whereas the developed countries like Africa, Central America, and Eastern Asia have the lowest rate of oral cavity cancer, for both males and females (Ferlay et al. 2010).

Oral cancer, with its widely variable rate of occurrence, has one of the highest incidences in Indian subcontinent where it ranks among the top three types of cancer in the country (Elango et al. 2006). Age-adjusted rate of oral cancer in India is high, that is, 20 per 100,000 population that accounts for over $30 \%$ of all cancerous persons in the country (Sankaranarayanan et al. 1927). It is of public health importance in India as it has been estimated that 83,000 new oral cancer cases occur here each year (Manoharan et al. 2004; Agrawal et al. 2012). The difficulty level is high because it is usually diagnosed at later stages which result in low treatment outcomes and considerable high cost to the patients who typically cannot afford this type of treatment (Khandekar et al. 2006). The prognosis for patients with oral cancer also remains poor in spite of advances in therapy of many other malignancies. Early diagnosis and treatment remains the key to improved patient survival. To achieve success in treatment, it is essential to determine the hidden patterns and trends in the oral disease data, which can be collected from oral healthcare industry and subsequently "analyzed or mined" to help healthcare practitioners for effective decisionmaking.

Manual extraction of patterns from the data has occurred for centuries. The Bayes' theorem (1700s) and regression 
analysis (1800s) were considered to be early methods of identifying patterns in the data. The proliferation, ubiquity and increasing power of computer technology have dramatically increased the data collection, storage, and manipulation ability (Han and Kamber 2012). As datasets have grown in size and complexity, the direct "hands-on" data analysis has increasingly been augmented with indirect, automated data processing, aided by other discoveries in computer science, such as neural networks, cluster analysis, genetic algorithms (1950s), decision trees (1960s) and support vector machines (1990s). Data mining is the process of applying these methods with the intention of uncovering hidden patterns in large datasets (Kantardzic and Mehmed 2003).

Data mining (the analysis step of the "knowledge discovery in databases" process, or KDD), an interdisciplinary subfield of computer science, is a computational process of discovering patterns in large datasets (Fayyad et al. 1996; Data Mining Curriculum (2006); Clifton and Christopher 2010; Hastie et al. 2009). It bridges the gap from applied statistics and artificial intelligence (which usually provide the mathematical background) to database management by exploiting the way the data are stored and indexed in the databases to execute the algorithms more efficiently, allowing such methods to be applied to ever larger datasets. The overall goal of data mining process is to extract information from a dataset and transform it into an understandable structure for further use (Data Mining Curriculum 2006). Apart from the raw analysis, it involves database and data management aspects, data preprocessing, model and inference considerations, interestingness metrics, complexity considerations, post-processing of discovered structures, visualization and online updating (Data Mining Curriculum 2006). There are various data mining tools available that can be used to predict behaviors and future trends, allowing businesses to make proactive, knowledge-driven decisions. Data mining tools can answer business questions that traditionally were too time consuming to resolve. They scour databases for hidden patterns, finding predictive information that experts may miss because it lies outside their expectations. The information gained can be used to develop a model for prediction and classification of new data (Cunningham and Holmes 1999).

The objective of this study is to develop various predictive data mining models and compare their performance in terms of survivability of oral cancer patients. The tool used for developing the data mining models is DTREG. DTREG (pronounced D-T-Reg) is a predictive modeling software that builds classification and regression decision trees, neural networks, support vector machine (SVM), GMDH polynomial networks, gene expression programs, $\mathrm{K}$-means clustering, discriminant analysis and logistic regression models that can describe data relationships. The
DTREG can be used to predict values for future observations and also has full support for time series analysis. It accepts a dataset in the form of table containing number of rows, whose columns represent attributes/variables. One of the variables is the "target variable" whose value is to be modeled and predicted as a function of the "predictor variables". The DTREG analyzes the data and generates a model showing how best it predicts the values of target variable based on the values of predictor variables (http:// www.dtreg.com).

The rest of the paper is organized as follows. Section 2 reviews the related literature and Sect. 3 covers the information about oral cancer. Section 4 discusses briefly the various predictive data mining models and Sect. 5 describes the various parameters to estimate the performance of models. In Sect. 6, experimental results are discussed and in Sect. 7, comparative performance of the models is given. Finally, Sect. 8 provides conclusion and future work.

\section{Literature survey}

Milovic and Milovic (2012) present the applicability of data mining in healthcare and explain how these patterns can be used by physicians to determine diagnoses, prognoses and apply treatments for patients in healthcare organizations. Anuradha and Sankaranarayanan (2012) have done a detailed survey on various methods adopted by the researchers for oral cancer detection at an earlier stage. A comparison is made among the various methods for identification and classification of cancers. Nahar et al. (2009) discuss to extract the significant prevention factors for particular type of cancer. To find out the prevention factors, they have first constructed a prevention factor dataset through an extensive literature review. Subsequently, three association rule-mining algorithms: Apriori, Predictive Apriori and Tertius algorithms have been employed to discover most of the significant prevention factors against a specific type of cancer. Experimental results illustrate that Apriori is the most useful association rule-mining algorithm for discovery of prevention factors.

Chuang et al. (2011) consider DNA repair genes. They chose single nucleotide polymorphisms (SNPs) dataset with 238 samples of oral cancer and control patients for disease prediction. All prediction experiments were conducted using the support vector machine and they reported that the performances of the holdout cross-validation were superior to tenfold cross-validation, and the best classification accuracy was $64.2 \%$. Gadewal and Zingde (2011) compiled and enlarged the oral cancer gene database to include 374 genes by adding 132 gene entries to enable fast retrieval of updated information. Kent (1996) has discussed 
a diagnosis method for oral cancer using genetic programming that can solve many complex problems. The genetic programming technique is used to evolve programs to diagnose oral cancer and pre-cancer in a sample of patients. Real data on the habits and lifestyles of patients are used to train and test the system. Despite inconsistencies in the input data, and a low proportion of positive samples, the evolved programs make accurate predictions and compete favorably with a previous neural network implementation of this problem.

Kaladhar et al. (2011) predict oral cancer survivability using classification algorithms that include CART, Random Forest, LMT and Naïve Bayesian classification algorithms. These algorithms classify the cancer survival using tenfold cross-validation and training data set. The Random Forest classification technique correctly classifies the cancer survival dataset leading to absolute relative error relatively less as compared to other methods. Sankaranarayanan (1990) reviews the epidemiologic and clinical aspects of the oral cancer in India. Causal association between oral cancer and the chewing of betel quids containing tobacco leaves or stem and other tobacco habits has extensively been studied. The exciting opportunity provided by the well-established oral precancerous lesions for intervention and early detection programs is also discussed. The peak age frequency of occurrence is at least a decade earlier than that described in Western literature. Sex ratio reveals a 2:1 preponderance of male patients. Only $10-15 \%$ of cases presented are in localized stage. The poor survival revealed by existing studies is mainly due to the overwhelming proportion of advanced cases. The excellent opportunity for more research and efforts in prevention and control of oral cancer in India is highlighted in this review.

Gupta and Misra (2013) outlined a metabolic model to grasp the cause impact connection and dependence interfaced with the perplexing collaborations of natural systems and atomic frameworks. The power for systemic tweaking conduct of thyroid hormone emission throughout the course of diverse time unit recreation is clarified in this study. Acquired effects demonstrate suitably the whole noticeable certainty of the medication impacts and are fit to continue according to the annoyances of the characteristic unit. Gupta et al. (2012) propose a methodology for recognizable proof and forecast of miRNA in infections through artificial neural networks (ANN). This thought utilizes both successive and structural characteristics of pre-miRNA to prepare the ANN for recognizable proof of miRNA in new viral genomes. The planned ANN was discovered with an exactness of $93.68 \%$ for the preparation dataset and $55.55 \%$ for the acceptance dataset. The outcomes demonstrate that this system might be utilized for distinguishing novel miRNA as a part of other viral genomes with respectable triumph. Abual-Rub et al.(2012) presents a hybrid harmony search algorithm (HHSA) as an intends to settle ab initio protein tertiary structure prediction problem. In HHSA, the iterated local search (ILS) is incorporated with the harmony.

\section{Oral cancer}

Oral cancer, a subtype of head and neck cancer, is a cancerous tissue growth located in the oral cavity (Werning 2007). It may arise as a primary lesion originating in any of the oral tissues, by metastasis from a distant site of origin, or by extension from a neighboring anatomic structure, such as the nasal cavity. The oral cancers may also originate in any of the tissues of the mouth. They may be of varied histologic types: teratoma, adenocarcinoma derived from a major or minor salivary gland, lymphoma from tonsillar or other lymphoid tissue, or melanoma from the pigment-producing cells of the oral mucosa. There are several types of oral cancers, but around $90 \%$ are squamous cell carcinomas (http://www.oralcancerfoundation. org/facts/index.htm), originating in the tissues that line the mouth and lips. Oral or mouth cancer most commonly involve the tongue. It may also occur on the floor of the mouth, cheek lining, gingiva (gums), lips, or palate (roof of the mouth). Most oral cancers look very similar under the microscope and they are called squamous cell carcinoma.

An oral cancer is caused by multiple factors, not single factor. Oral malignancy is most likely caused by a combination of extrinsic and intrinsic factors acting in concert over a period of time. Symptoms demonstrate that there is at least a contributing component related to a genetic susceptibility of the individual exposed to carcinogens and a potential for malignant transformation of the oral tissue. The epidemiologic data indicate that a strong correlation exists between the exposure to many potential carcinogens and the increased risk for development of an oral cancer following long exposure or early exposure to these carcinogens. Most published reports indicate that the age, gender, race, tobacco use, alcohol use (especially tobacco and alcohol in combination), presence of a synchronous cancer of the upper aero-digestive tract, poor nutritional status, infection with certain viruses, oral lichen planus and immune deficiencies all increase the relative risk for developing an oral cancer.

Historically, this has been attributed to widespread usage of smokeless tobacco, an established carcinogen. Well-supported by the evidence, great emphasis is being laid by the government and non-government organizations toward effective tobacco control. Over last several decades, billions of rupees have been spent to educate the public, implement laws effectively, rehabilitate tobacco growers, 
build cessation facility, create health infrastructure, etc., to reduce the smokeless tobacco usage. However, oral cancer that is an uncommon disease in the west continues to be the major cause of cancer death in Indian population. About 2,000 deaths a day in India is tobacco-related (http://www. oralcancerawareness.org).

The symptoms for an oral cancer at an earlier stage (Scully et al. 2008) are: (a) patches inside the mouth or on lips that are white, red, or mixture of white and red, (b) any sore or area in the mouth which does heel for discolored more than 14 days, (c) bleeding in the mouth, (d) difficulty or pain when swallowing, and (e) a lump in the neck. These symptoms identify the suspect for a cancer. Treatments for oral cancers include surgery, radiation therapy and chemotherapy (http://www.yourtotalhealth.ivillage.com). But even that is not always successful as $70 \%$ of the cases after treatment lead to relapse and the result is death. The treatment is successful only if the lesion is diagnosed early, but sadly many times, it is ignored and the patient reports when the lesion has spread so much that the treatment is impossible or even if done the long-term prognosis is poor. Prognosis of oropharyngeal squamous cell carcinoma (SCC) (oral cavity and pharynx) depends on early diagnosis, despite advanced surgical techniques and adjuvant treatment, the 5-year survival rate remains $\sim 40-50 \%$ (Jemal et al. 2002; Woolgar et al. 1995). The treatment cost is 3.5 lakh and in spite of this cost there is no guarantee that it will surely be cured. The surgery needs cutting half the face and tongue and extending to the neck and the patient looks horrible after surgery.

\section{Predictive data mining models}

Data Mining is an analytic process designed to explore data in search for consistent patterns and/or systematic relationships between variables, and then to validate the findings by applying the detected patterns to new subsets of data. The ultimate goal of data mining is prediction and the predictive data mining is the most common type of data mining and it has the most direct business applications. Predictive modeling is the process by which a model is created or chosen to try to best predict the probability of an outcome (Geisser et al. 1993). The process of data mining consists of three stages: (a) the initial exploration, (b) model building or pattern identification with validation/ verification and (c) deployment (i.e., the application of the model to new data to generate predictions).

\subsection{Decision tree model}

A decision tree is a logical model represented as a binary (two-way split) tree that shows how the value of a target variable can be predicted using the values of a set of predictor variables. The nodes in the tree are denoted by rectangular boxes. Each node represents a set of records (rows) from the original dataset. A node is called interior node or leaf node depending on it has child node or not. The topmost node, called the root node, represents all the rows in the dataset. A decision tree is constructed by a binary split that divides the rows in a node into two groups (child nodes). The same procedure is then used to split the child groups. This process is called recursive partitioning. The split is selected to construct a tree that can be used to predict the value of the target variable.

\subsection{TreeBoost model}

Boosting is a technique for improving the accuracy of a predictive function by applying the function repeatedly in a series and combining the output of each function with weight so that the total error of the prediction is minimized. In many cases, the predictive accuracy of such a series greatly exceeds the accuracy of the base function used alone. Mathematically, a TreeBoost model can be described as:

Predicted Target $=F_{0}+B_{1} \times T_{1}(X)+B_{2} \times T_{2}(X)+\cdots$

$$
+B_{M} \times T_{M}(X),
$$

where $F_{0}$ is the starting value for the series (the median target value for a regression model), $X$ a vector of pseudoresidual values remaining at this point in the series, $T_{1}(X)$, $T_{2}(X), \ldots, T_{M}(X)$ trees fitted to the pseudo-residuals and $B_{1}$, $B_{2}, \ldots, B_{M}$ coefficients of the tree node predicted values that are computed by the TreeBoost algorithm.

The first tree is fitted to the data. The residuals (error values) from the first tree are then fed into the second tree which attempts to reduce the error. This process is repeated through a series of successive trees. The final predicted value is formed by adding the weighted contribution of each tree.

\subsection{Decision tree forest model}

A decision tree forest consists of an ensemble (collection) of decision trees whose predictions are combined to make the overall prediction for the forest. A decision tree forest is similar to a TreeBoost model in the sense that a large number of trees are grown. However, the TreeBoost generates a series of trees with the output of one tree going into the next tree in the series. In contrast, a decision tree forest grows a number of independent trees in parallel, and they do not interact until all of them have been built. Both TreeBoost and decision tree forests produce high accuracy models. Experiments have shown that the TreeBoost works 
better with some applications and decision tree forests with others; so it is better to try both methods and compare the results.

\section{Estimation of performance of data mining models}

Assessing the performance of a data mining technique is an essential part of machine learning. Evaluation strategy is a yardstick to analyze the productivity and execution of any model. The evaluation is imperative for comprehension of the nature of the model, for refining parameters in the iterative process of learning and for selecting the most adequate model.

\subsection{Misclassification table}

If the target variable is categorical and a classification tree is build, then a misclassification summary table presents the number of rows with a particular category that have been misclassified by the tree. There are two sections in the table: one for misclassifications for the training dataset and one for misclassification for the validation data (either cross-validation or random-holdback rows).

\subsection{Confusion matrix}

The confusion matrix provides detailed information about how the data rows are classified by the model. The matrix has a row and column for each category of the target variable. The categories in the first column are the actual categories of the target variable. The categories shown across the top of the table are the predicted categories. The numbers in cells are the weights of the data rows with the actual category of the row and the predicted category of the column. The numbers in diagonal cells are the weights for the correctly classified cases where the actual category matches the predicted category. The off-diagonal cells have misclassified row weights.

\subsection{Sensitivity and specificity}

The sensitivity refers to the probability that the algorithms can correctly predict nonmalignancy. The specificity refers to the probability that the algorithms can correctly predict malignant. The sensitivity and specificity report is generated only for the classification problems (categorical target variable). One category of the target variable is called the positive category, and the other is called the negative category. True positive (TP) means that the patients who are predicted as malignant among the malignant patients. True negative (TN) means that the patients who are predicted as nonmalignant among nonmalignant patients. False positive
(FP) means that the patients who are predicted as nonmalignant among malignant patients. False negative (FN) means that the patients who are predicted as malignant among nonmalignant patients. The sensitivity and specificity have been calculated by TP, TN, FP and FN as shown in Table 1.

\subsection{Probability calibration report}

The probability calibration report shows how the predicted probability of a target category is distributed and provides a means for gauging the accuracy of predicted probabilities. The probability calibration report is generated only when a classification analysis is performed and there are two target categories.

\subsection{Probability threshold report}

The probability threshold report provides the information about how different probability thresholds would affect target category assignments. The threshold report provides a convenient way to see the trade-off between the impurity and loss as the probability threshold is varied. The probability threshold report is generated only when a classification analysis is performed and there are two target categories.

\subsection{Lift and gain table}

The lift and gain table is a useful tool for measuring the value of a predictive model. The lift and gain values are especially useful when a model is used to target (prioritize) marketing efforts. The basic idea of lift and gain is to sort the predicted target values in decreasing order of purity on some target category and then compare the proportion of the cases with the category in each bin with the overall proportion. In case of a model with a continuous target variable, the predicted target values are sorted in decreasing target value order and then compared with the mean target value. The lift and gain values show how much improvement the model provides in picking out the best 10 , $20 \%$, etc. of the cases.

Table 1 Sensitivity and Specificity

\begin{tabular}{lll}
\hline Actual class & Predicted class & \\
\cline { 2 - 3 } & True & False \\
\hline True & TP & FP \\
False & FN & TN \\
& TP + FN & FP + TN \\
\hline Sensitivity $=\mathrm{TP} /(\mathrm{TP}+\mathrm{FN})$ & & \\
Specificity $=\mathrm{TN} /(\mathrm{FP}+\mathrm{TN})$ & &
\end{tabular}




\section{Experimental results}

The complete process followed to create the database of oral cancer patients has been presented in the previous paper "Framework for early detection and prevention of oral cancer using data mining" published by the authors. The database is further stored in csv file format so that it can be used to build various models using DTREG tool (Neha and Om 2012). The number of variables (data columns) is 33 as shown in Table 2. All data rows are used for data subsetting. The number of data rows is 1,024 and the total weight for all rows is 1,024 . There are no rows with missing target or weight values. However, there is only one row with missing predictor value.

\subsection{Building a model}

There are three prediction models that have been developed using classification technique and there are certain commonalities among all the models. All the models have considered only 19 predictor variables and 01 target variable i.e., 'survival'. The classification technique is used for analysis and the category weights are distributed over entire data file. The misclassification costs are equal (unitary) and the variable weights are also equal.

\subsubsection{Single tree model}

Maximum splitting levels of single tree model is 10 . Gini is the splitting algorithm. Minimum size node to split is 10 , whereas the minimum rows allowed in a node are 5 .
Maximum categories for continuous predictors are 1,000. Cross-validation method with tenfolds is used for tree pruning and validation. Minimum cost complexity $(0.00$ $\mathrm{SE})$ is used as tree pruning criterion.

\subsubsection{Decision tree forest model}

Maximum trees in decision tree forest are 200. Maximum splitting levels are 50. Minimum size node to split is 2 . Maximum categories for continuous predictors are 1,000. Surrogate splitters are used for missing values. Out of Bag (OOB) tree validation method is used.

\subsubsection{TreeBoost model}

Maximum trees in TreeBoost series are 400. Maximum splitting levels are 5. Minimum size node to split is 10 . Maximum categories for continuous predictors are 1,000. Random sampling (20\%) validation method is used. Tree pruning criterion is minimum absolute error.

\subsection{Model size}

\subsubsection{Single tree}

Maximum depth of the tree is 2. Total number of group splits is 1 . The full tree has two terminal nodes. The minimum validation relative error occurs with two nodes. The relative error value is 0.0000 with a standard error of 0.0000 .

Table 2 Variables and categories

\begin{tabular}{rllll}
\hline No. & Variable & Categories & No. & Variable \\
\hline 1 & Case Id & - & 17 & Diagnosis \\
2 & Clinical symptoms & 4 & 18 & Staging \\
3 & History of addiction & 3 & 19 & Surgery \\
4 & History of addiction 1 & - & 20 & Radiotherapy \\
5 & Comorbid Condition & 2 & 21 & Chemotherapy \\
6 & Comorbid Condition 1 & - & 22 & Histopathology \\
7 & Gross examination & 5 & 23 & 1st follow-up symptoms \\
8 & Site & 4 & 24 & 1st follow-up examination \\
9 & Predisposing factor & 4 & 25 & 2nd follow-up symptoms \\
10 & Tumor size & 3 & 26 & 2nd follow-up examination \\
11 & Neck node & 2 & 27 & 3rd follow-up symptoms \\
12 & LFT & 2 & 28 & 3rd follow-up examination \\
13 & FNAC of neck node & 2 & 29 & 4th follow-up symptoms \\
14 & Diagnostic biopsy & 3 & 30 & 4th follow-up examination \\
15 & USG & 2 & 31 & 5th follow-up symptoms \\
16 & CT scan/MRI & 2 & 32 & 5th follow-up examination
\end{tabular}




\subsubsection{Decision tree forest}

The full forest has 200 trees. Four predictors (out of 18) have been used for each split. Maximum depth of any tree in the forest is 4 . Average number of group splits in each tree is 1.5 .

\subsubsection{TreeBoost}

All 18 predictors have been considered for each split. The full series has 400 trees. The minimum error with the training data occurs with 30 trees. The minimum error with the test data also occurs with 30 trees. The minimum point is smoothed by five trees. The specified minimum number of trees is 10 . The tree series has been pruned to 30 trees. Maximum depth of any tree in the series is 2. Average number of group splits in each tree is 1.0.

\subsection{Misclassification table}

Misclassification statistics is same for single tree for both training and validation data as well as for decision tree forest for out of bag validation data, as shown in Table 3 . The misclassification statistics of TreeBoost model for both training and validation data is shown in Tables 4 and 5, respectively. However, overall accuracy for all the models is $100.00 \%$.

\subsection{Confusion matrix}

The confusion matrix for both training and validation data for single tree and decision tree forest model is same and it is shown in Table 6. The confusion matrix of TreeBoost

Table 3 Misclassification for single tree, decision tree forest

\begin{tabular}{|c|c|c|c|c|c|c|}
\hline \multirow[t]{2}{*}{ Category } & \multicolumn{2}{|l|}{ Actual } & \multicolumn{4}{|c|}{ Misclassified } \\
\hline & Count & Weight & Count & Weight & Percent & Cost \\
\hline A & 333 & 333 & 0 & 0 & 0.000 & 0.000 \\
\hline $\mathrm{D}$ & 691 & 691 & 0 & 0 & 0.000 & 0.000 \\
\hline Total & 1,024 & 1,024 & 0 & 0 & 0.000 & 0.000 \\
\hline
\end{tabular}

Table 4 Misclassification for TreeBoost model (training data)

\begin{tabular}{llllllll}
\hline Category & \multicolumn{2}{l}{ Actual } & & & \multicolumn{3}{c}{ Misclassified } \\
\cline { 2 - 3 } & Count & Weight & & Count & Weight & Percent & Cost \\
\hline A & 266 & 266 & 0 & 0 & 0.000 & 0.000 \\
D & 553 & 553 & & 0 & 0 & 0.000 & 0.000 \\
Total & 819 & 819 & & 0 & 0 & 0.000 & 0.000 \\
\hline
\end{tabular}

model for both training and validation data is shown in Table 7 .

\subsection{Sensitivity and specificity}

'Survival $=\mathrm{D}$ ' is considered as a positive and 'Survival $=A$ ' is considered as negative for all the models developed in this work. The positive/negative ratio, accuracy, true positive (TP), true negative (TN), false positive (FP), false negative (FN), sensitivity, specificity, geometric mean of sensitivity and specificity, positive predictive value (PPV), negative predictive value (NPV) and geometric mean of PPV and NPV for training and validation data for all the models developed are shown in Table 8.

\subsection{Probability calibration}

Probability calibration is done for Survival $=$ Dead (D) as well as Survival $=$ Alive $(\mathrm{A})$ for all the models.

\subsubsection{Single tree}

Probability calibration for Survival $=\mathrm{D}$ or A is same. The average weighted probability error for training

Table 5 Misclassification for TreeBoost model (validation data)

\begin{tabular}{lccllllll}
\hline Category & Actual & & & \multicolumn{2}{l}{ Misclassified } \\
\cline { 2 - 3 } & Count & Weight & & Count & Weight & Percent & Cost \\
\hline A & 67 & 67 & & 0 & 0 & 0.000 & 0.000 \\
D & 138 & 138 & & 0 & 0 & 0.000 & 0.000 \\
Total & 205 & 205 & & 0 & 0 & 0.000 & 0.000 \\
\hline
\end{tabular}

Table 6 Confusion matrix (single tree and decision tree forest)

\begin{tabular}{|c|c|c|c|c|}
\hline \multirow[t]{3}{*}{ Actual Category } & \multicolumn{2}{|c|}{ Training data } & \multicolumn{2}{|c|}{ Validation data } \\
\hline & \multicolumn{2}{|c|}{ Predicted category } & \multicolumn{2}{|c|}{ Predicted category } \\
\hline & A & $\mathrm{D}$ & A & $\mathrm{D}$ \\
\hline A & 333 & 0 & 333 & 0 \\
\hline D & 0 & 691 & 0 & 691 \\
\hline
\end{tabular}

Table 7 Confusion matrix (TreeBoost)

\begin{tabular}{|c|c|c|c|c|}
\hline \multirow[t]{3}{*}{ Actual Category } & \multirow{2}{*}{\multicolumn{2}{|c|}{$\frac{\text { Training data }}{\text { Predicted category }}$}} & \multirow{2}{*}{\multicolumn{2}{|c|}{$\frac{\text { Validation data }}{\text { Predicted category }}$}} \\
\hline & & & & \\
\hline & A & $\mathrm{D}$ & A & $\mathrm{D}$ \\
\hline A & 266 & 0 & 67 & 0 \\
\hline D & 0 & 533 & 0 & 138 \\
\hline
\end{tabular}


Table 8 Sensitivity and specificity

\begin{tabular}{|c|c|c|c|}
\hline & \multirow{2}{*}{$\begin{array}{l}\text { Single tree, } \\
\text { decision tree } \\
\text { forest (training, } \\
\text { validation) }\end{array}$} & \multicolumn{2}{|c|}{ TreeBoost model } \\
\hline & & $\begin{array}{l}\text { Training } \\
\text { data }\end{array}$ & $\begin{array}{l}\text { Validation } \\
\text { data }\end{array}$ \\
\hline Total records & 1,024 & 819 & 205 \\
\hline Positive/negative ratio & 2.08 & 2.08 & 2.08 \\
\hline Accuracy & $100 \%$ & $100 \%$ & $100 \%$ \\
\hline True positive (TP) & $691(67.5 \%)$ & $\begin{array}{l}553 \\
\quad(67.5 \%)\end{array}$ & $\begin{array}{l}138 \\
\quad(67.5 \%)\end{array}$ \\
\hline True negative $(\mathrm{TN})$ & $333(32.5 \%)$ & $\begin{array}{l}266 \\
\quad(32.5 \%)\end{array}$ & $\begin{array}{l}67 \\
(32.5 \%)\end{array}$ \\
\hline False positive (FP) & $0(0.0 \%)$ & $0(0.0 \%)$ & $0(0.0 \%)$ \\
\hline False negative (FN) & $0(0.0 \%)$ & $0(0.0 \%)$ & $0(0.0 \%)$ \\
\hline Sensitivity & $100 \%$ & $100 \%$ & $100 \%$ \\
\hline Specificity & $100 \%$ & $100 \%$ & $100 \%$ \\
\hline $\begin{array}{l}\text { Geometric mean of } \\
\text { sensitivity and } \\
\text { specificity }\end{array}$ & $100 \%$ & $100 \%$ & $100 \%$ \\
\hline $\begin{array}{l}\text { Positive predictive } \\
\text { value (PPV) }\end{array}$ & $100 \%$ & $100 \%$ & $100 \%$ \\
\hline $\begin{array}{l}\text { Negative predictive } \\
\text { value (NPV) }\end{array}$ & $100 \%$ & $100 \%$ & $100 \%$ \\
\hline $\begin{array}{l}\text { Geometric mean of } \\
\text { PPV and NPV }\end{array}$ & $100 \%$ & $100 \%$ & $100 \%$ \\
\hline Precision & $100 \%$ & $100 \%$ & $100 \%$ \\
\hline
\end{tabular}

data $=0.000000$ and that for the training data $=0.000000$. The average weighted probability error for validation data $=0.000000$ and that for the validation data $=0.000000$.

\subsubsection{Decision tree forest}

In this model also, the probability calibration for Survival $=\mathrm{D}$ or $\mathrm{A}$ is same. The average weighted probability error for training data $=0.000042$ and that the average weighted squared probability error for training data $=0.000073$.

\subsubsection{TreeBoost}

Probability calibration for Survival = D or A is same for this model as well. The average weighted probability error for training data $=0.324473$ and the average weighted squared probability error for training data $=0.346345$. The average weighted probability error for validation data $=0.008016$ and the average weighted squared probability error for validation data $=0.008553$.

\subsection{Probability threshold}

Probability threshold analysis is carried out for Survival $=\mathrm{D}$ for all the models.

\subsubsection{Single tree}

Area under ROC curve (AUC) for training and test data $=1.000000$. Threshold to minimize misclassification for both training and test data $=0.000000$. Threshold to minimize weighted misclassification for both training and test data $=0.000000$.

\subsubsection{Decision tree forest}

Area under ROC curve $(\mathrm{AUC})=1.000000$. Threshold to minimize misclassification $=0.028571$. Threshold to minimize weighted misclassification $=0.028571$. Threshold to balance misclassifications $=0.021429$.

\subsubsection{TreeBoost}

Area under ROC curve (AUC) for training data $=1.000000$. Threshold to minimize misclassification for training data $=0.499121$. Threshold to minimize weighted misclassification for training data $=0.499121$. Threshold to balance misclassifications for training data $=0.249561$.

Area under ROC curve (AUC) for test data $=1.000000$. Threshold to minimize misclassification for test data $=0.012298$. Threshold to minimize weighted misclassification for test data $=0.012298$. Threshold to balance misclassifications for test data $=0.006149$.

\subsection{Lift and gain}

\subsubsection{Single tree and decision tree forest}

Lift and gain for training data as well as validation data for both the models are same:

Lift/gain for Survival $=\mathrm{A}$

Average gain $=2.012$

Percent of cases with Survival = A: $32.52 \%$

Lift/gain for Survival = D

Average gain $=1.366$

Percent of cases with Survival = D: $67.48 \%$

\subsubsection{TreeBoost}

Lift and gain for training data is shown below:

Lift/gain for Survival $=\mathrm{A}$ 
Average gain $=2.018$

Percent of cases with Survival = A: $32.48 \%$

Lift/gain for Survival = D

Average gain $=1.367$

Percent of cases with Survival = D: $67.52 \%$

Lift and gain for validation data is shown below:

Lift/gain for Survival = A

Average gain $=1.990$

Percent of cases with Survival = A: $32.68 \%$

Lift/gain for Survival = D

Average gain $=1.361$

Percent of cases with Survival = D: $67.32 \%$

6.9 Overall importance of variable

\subsubsection{Single tree}

Variable

Diagnostic biopsy

6.9.2 Decision tree forest

\begin{tabular}{lc}
\hline Variable & Importance \\
\hline Diagnostic biopsy & 100.000 \\
Diagnosis & 65.210 \\
Radiotherapy & 50.881 \\
Gross examination & 41.033 \\
Histopathology & 38.369 \\
Clinical symptom & 25.738 \\
Staging & 25.238 \\
Neck nodes & 13.800 \\
Chemotherapy & 11.243 \\
Predisposing factor & 6.072 \\
Tumor size & 4.863 \\
USG & 4.062 \\
LFT & 2.510 \\
Co Morbid condition & 2.142 \\
Site & 1.393 \\
History of addiction & 0.985 \\
\hline
\end{tabular}

\subsubsection{TreeBoost}

\begin{tabular}{lc}
\hline Variable & Importance \\
\hline Diagnostic biopsy & 100.000 \\
Radiotherapy & 68.433 \\
Histopathology & 4.371 \\
\hline
\end{tabular}

\section{Comparative performance of models}

The attempt has been made to compare all the models to identify the effectiveness of the models to predict the survivability of the oral cancer patients. Initially, the comparison has been made on the basis of various criteria used to develop each one of them and it is presented in Table 9 .

Further, comparison of all the models on the basis of performance is discussed.

Misclassification: There is no row in any category which is misclassified by the tree in any of the models.

Confusion matrix: All the cases have been correctly classified and the actual category matches with the predicted category. The off-diagonal cells have ' 0 ', which indicates that there is no misclassified row in any of the models

Table 9 Comparison the model development criteria

\begin{tabular}{|c|c|c|c|c|}
\hline $\begin{array}{l}\text { Sr. } \\
\text { no. }\end{array}$ & Criteria & Single tree & $\begin{array}{l}\text { Decision } \\
\text { tree forest }\end{array}$ & TreeBoost \\
\hline 1 & Maximum trees & 01 & 200 & 400 \\
\hline 2 & $\begin{array}{l}\text { Maximum } \\
\text { splitting levels }\end{array}$ & 10 & 50 & 05 \\
\hline 3 & $\begin{array}{r}\text { Minimum size } \\
\text { node to split }\end{array}$ & 10 & 0210 & \\
\hline 4 & $\begin{array}{l}\text { Maximum } \\
\text { categories for } \\
\text { continuous } \\
\text { predictors }\end{array}$ & 1,000 & 1,000 & 1,000 \\
\hline 5 & $\begin{array}{c}\text { Validation } \\
\text { method }\end{array}$ & $\begin{array}{l}\text { Cross- } \\
\text { validation } \\
\text { with } \\
\text { tenfolds }\end{array}$ & $\begin{array}{l}\text { Out of bag } \\
\text { tree } \\
\text { validation }\end{array}$ & $\begin{array}{l}\text { Random } \\
\text { Sampling } \\
(20 \%) \\
\text { validation }\end{array}$ \\
\hline 6 & $\begin{array}{l}\text { Tree pruning } \\
\text { criteria }\end{array}$ & $\begin{array}{l}\text { Minimum } \\
\text { cost } \\
\text { complexity }\end{array}$ & - & $\begin{array}{l}\text { Minimum } \\
\text { absolute } \\
\text { error }\end{array}$ \\
\hline
\end{tabular}


Sensitivity and specificity: It is $100 \%$ for all the models.

Probability calibration: Comparison of probability calibration for Survival $=$ D or A for all the models on training data as well as validation data shows that the 'Average Weighted Probability Error' and 'Average Weighted Squared Probability Error' are slightly higher in the TreeBoost model.

Threshold analysis: Comparison of probability threshold analysis for Survival = D on training data as well as validation data shows that the Area under ROC curve is 1.000000 for all the models. However, 'Threshold to minimize the misclassification', 'Threshold to minimize the weighted misclassification' and 'Threshold to balance misclassification' is marginally higher in the TreeBoost model.

Lift and gain: Average gain and percentage of cases with Survival = A or D is slightly better in the case of TreeBoost model in comparison to other models.

Importance of variable: The variable 'Diagnostic Biopsy' is the most important variable according to all the models. However, the variable 'Diagnosis' is the next important variable as per Decision Tree Forest model, whereas the variable 'Radiotherapy' is the next important variable as per the TreeBoost model.

Analysis run time: 00:00.40, 00:00.56 and 00:01.24 are the run times for single tree, decision tree forest and TreeBoost, respectively.

\section{Conclusion and future work}

All the models built for predicting the survivability of oral cancer patients show similar results and performance. However, the TreeBoost model is marginally better than the others as all 18 predictors are considered for each spit. The experimental result of probability calibration, Threshold analysis and lift-gain are also slightly better in the TreeBoost model. Thus, the TreeBoost classification model is effective for determining the survival rate of oral cancer patients. Our future work shall involve building neural network models and comparing them for accessing the survivability of oral cancer patients.

Acknowledgments The authors would like to thank Dr. Vijay Sharma, MS, ENT, for his valuable contribution in understanding the occurrence and diagnosis of Oral Cancer. The authors express their sincere thanks to the management and staff of Indian School of Mines, for their constant support and motivation.

\section{References}

Abual-Rub MS (2012) et al A hybrid harmony search algorithm for ab initio protein tertiary structure prediction. Netw Model Anal Health Inform Bioinform 1(3):69-85
Agrawal M, Pandey S, Jain S, Maitin S (2012) Oral cancer awareness of the general public in Gorakhpur City, India. Asian Pac J Cancer Prev 13:5195-5199

Anuradha K, Sankaranarayanan K (2012) Identification of suspicious regions to detect oral cancers at an earlier stage-a literature survey. Int J Adv Eng Technol 3(1):84-91

Christopher C (2010) Encyclopaedia Britannica: definition of data mining. Retrieved 2010-12-09

Chuang L-Y, Wu K-C, Chang H-W, Yang C-H (2011) Support vector machine-based prediction for oral cancer using four snps in DNA repair genes. In: Proceedings of the international multiconference of engineers and computer scientists, March $16-182011$

Cunningham SJ, Holmes G (1999) Developing innovative applications in agriculture using data mining. In: Proceedings of the Southeast Asia regional computer confederation conference, 1999

Data Mining Curriculum (2006) ACM SIGKDD. 2006-04-30. Retrieved 2011-10-28

Elango JK, Gangadharan P, Sumithra S, Kuriakose MA (2006) Trends of head and neck cancers in urban and rural India. Asian Pac J Cancer Prev 7(1):108-112 (view at Scopus)

Fayyad UM, Piatetsky-Shapiro G, Smyth P (1996) From data mining to knowledge discovery: an overview. In: Advances in knowledge discovery and data mining. AAAI Press, Menlo Park

Ferlay J, Shin HR, Bray F et al (2010) Estimates of worldwide burden of cancer in 2008: GLOBOCAN 2008. Int J Cancer 12:2893-2917

Gadewal NS, Zingde SM (2011) Database and interaction network of genes involved in oral cancer: Version II. Bioinformation 6(4):169-170

Gupta MK, Misra K (2013) Modeling and simulation analysis of propyl-thiouracil (PTU), an anti-thyroid drug on thyroid peroxidase (TPO), thyroid stimulating hormone receptor (TSHR), and sodium iodide (NIS) symporter based on systems biology approach. Netw Model Anal Health Inform Bioinform 2(1):45-57

Gupta MK et al (2012) Prediction of miRNA in HIV-1 genome and its targets through artificial neural network: a bioinformatics approach. Netw Model Anal Health Inform Bioinform 1(4):141-151

Han J, Kamber M (2012) Data mining: concepts and techniques, 3rd edn. Morgan Kaufmann

Jemal A, Thimas A, Murray T, Thun M (2002) Cancer statistics, 2002. CA Cancer J Clin 52:181-182

Jemal A, Siegel R, Xu J, Ward E (2010) Cancer statistics. CA Cancer J Clin 60:277-300

Kaladhar DSVGK, Chandana B, BharathKumar P (2011) Predicting cancer survivability using classification algorithms. Int J Res Rev Comput Sci (IJRRCS) 2(2):340-343

Kent S (1996) Diagnosis of oral cancer using genetic programminga technical report, CSTR -96-14

Khandekar PS, Bagdey PS, Tiwari RR (2006) Oral cancer and some epidemiological factors: a hospital based study. Indian J Community Med 31(3):157-159

Manoharan N, Tyagi BB, Raina V (2010) Cancer incidences in rural Delhi-2004-2005. Asian Pac J Cancer Prev 11(1):73-78 (view at Scopus)

Mehmed K (2003) Data mining: concepts, models, methods, and algorithms. Wiley, Chichester (ISBN 0-471-22852-4. OCLC 50055336)

Milovic B, Milovic M (2012) Prediction and decision making in health care using data mining. Int $\mathrm{J}$ Public Health Sci 1(2):69-78

Nahar J et al (2009) Significant cancer prevention factor extraction: an association rule discovery approach. J Med Syst. doi:10.1007/ s10916-009-9372-8 
Neha S, Om H (2012) Framework for early detection and prevention of oral cancer using data mining. Int $\mathrm{J}$ Adv Eng Technol 4(2):302-310

Sankaranarayanan R (1990) Oralcancer in India: an epidemiologic and clinical review. Oral Surg Oral Med Oral Pathol 69(3):325-330

Sankaranarayanan R, Ramadas K, Thomas G et al (2005) Effect of screening on oral cancer mortality in Kerala, India: a clusterrandomised controlled trial. Lancet 365(9475):1927-1933 (view at Publisher View at Google Scholar View at Scopus)

Scully C, Bagan JV, Hopper C, Epstein JB (2008) Oral Cancer: current and future diagnostics techniques-a review article. Am J Dent 21(4):199-209
Seymour G (1993) Predictive inference: an introduction. Chapman \& Hall, New York (ISBN 0-412-03471-9)

Trevor H, Robert T, Jerome F (2009) The elements of statistical learning: data mining, inference, and prediction. Retrieved 2012-08-07

Werning JW (2007) Oral cancer: diagnosis, management, and rehabilitation. Thieme, New York

Woolgar JA, Scott J, Vaughan ED, Brown JS, West CR, Rogers S (1995) Survival, metastasis and recurrence of oral cancer in relation to pathological features. Ann R Coll Surg Engl $77: 325-331$ 